\title{
Energy consumption index minimized resource allocation in hybrid energy multiuser OFDM system with distributed antennas
}

\author{
Huanhuan $\mathrm{MAO}^{1}$, Pengcheng $\mathrm{Zhu}^{1, *}$, and Jiamin $\mathrm{Li}^{1}$ \\ ${ }^{1}$ National Mobile Communications Research Laboratory, Southeast University, Nanjing, 210096, P. \\ R. China
}

\begin{abstract}
Energy harvesting is one of the promising option for realization of green communication and has been a growing concern recently. In this paper, we address the downlink resource allocation in OFDM system with distributed antennas with hybrid power supply base station, where energy harvesting and non-renewable power sources are used complementarily. A joint subcarrier and power allocation problem is formulated for minimizing the net Energy Consumption Index (ECI) with system Quality of Service (QoS) and bit error rates constraint. The problem is a 0-1 mixed integer nonlinear programming problem due to the binary subcarrier allocation variable. To solve the problem, we design an algorithm based on Lagrange relaxation method and fraction programming which optimizes the power allocation and subcarrier allocation iteratively in two nests. Simulation results show that the proposed algorithm converges in a small number of iterations and can improve net ECI of system greatly.
\end{abstract}

\section{Introduction}

In the past decade, green communication has become an unignorable and urgent topic because of the enormous energy consumption in mobile communication systems. Only for radio frequency energy, it is up to $27 \mathrm{GW}$ in 2015 [1] and will continuously increase. This trend has stimulated growing interest of researchers on innovative energy supply schemes, such as energy harvesting. With energy harvesting, renewable energy such as wind, solar and thermal replaces the conventional nonrenewable energy sources, which in turn reduces the emissions of carbon dioxide and other toxic gas. However, due to the intermittent and random nature of energy harvesting, it is instable and is usually used as an auxiliary of nonrenewable energy sources such as power grids and diesel engines. The nature of energy harvesting makes it challenging to derive transmission schemes in existing systems [2]. Various aspects of resource allocation scheme have been studied in energy harvesting. In a multi-picocell downlink network [3], power allocation for maximizing the energy efficient was studied and an offline scheme which needs the non-causal CSI was proposed. For cellular network, there have been significant research progress in resource allocation of various types. In [4], energy efficient resource allocation for OFDMA systems with hybrid

\footnotetext{
* Corresponding author: p.zhu@seu.edu.cn
} 
power supply base station was studied. An offline scheme with non-causal CSI and a suboptimal online scheme were proposed to jointly allocate the power and subcarrier. In [5], an OFDM-based cellular system was studied, and a low complexity suboptimal resource allocation algorithm was proposed. The algorithm assigns subcarrier based on channel power gain and performs power allocation separately.

Distributed antenna system (DAS), has been proposed as a promising solution for future mobile communications due to its advantages of improving the spectral efficiency and power efficiency [6]. Resource allocation in cellular DAS system has been investigated by many researchers [7] [8]. The authors in [7] investigated energy efficient resource allocation considering proportional fairness for a downlink multiuser OFDM system with distributed antennas. A low complexity suboptimal algorithm which separate the subcarrier and power allocations was proposed. In [8], maximize rate-sum capacity resource allocation problem was derived in OFDM distributed antenna systems and the performances was compared between co-located systems (CAS) and DAS.

In this paper, we focus on a OFDM system with distributed antennas with a hybrid power supply base station. Assuming perfect CSI at base station, we define the ratio of nonrenewable power and sun rate a net Energy Consumption Index(ECI) and minimize it under the system Quality of Service(QoS) and bit error rates constraints by jointly optimizing the subcarrier and power allocation. The problem is a 0-1 mixed integer nonlinear programming problem. In order to solve this problem, we propose a two nesting iterative algorithm based on fraction programming and Lagrangian relaxation method. For the outer nest, Dinkelbach method is adopted to ensure the convergent net ECI. And the inner nest derives a resource allocation policy. First, the subcarrier allocation variable is relaxed and the original problem becomes a convex problem. Then the Lagrange dual function of the relaxed problem and the optimal power allocation policy are derived. Simulation results show the proposed algorithm converges quickly and significant improvements on system net ECI is derived.

\section{System model}

\subsection{Channel model}

Consider the downlink of an OFDM-DAS system with M remote antenna units (RAUs) with single antenna, $\mathrm{N}$ subcarriers and $\mathrm{K}$ single antenna users, as illustrated in Fig.1. The central RAU is a base station with a hybrid renewable and non-renewable power supply which connecting other RAUs via fiber-optical and plays a central processing unit role. The signal received by user $\mathrm{k}$ at subcarrier $\mathrm{n}$ from RAU $\mathrm{m}$ can be expressed as

$$
y_{k, n, m}=\xi_{k, n, m} h_{k, n, m} \sqrt{p_{k, n, m}} x_{k, n, m}+z_{k, n, m},
$$

where $h_{k, n, m}$ is the channel fading from RAU $\mathrm{m}$ to user $k$ on subcarrier $n$, $z_{k, n, m} \sim \mathrm{CN}\left(0, \sigma_{z}^{2}\right)$ refers to the additive Gaussian white noise (AGWN), $x_{k, n, m}$ and $p_{k, n, m}$ represent the information symbol and transmit power on subcarrier $n$ to user $k$ from RAU $m$, and $\xi_{k, n, m}$ stands for a binary subcarrier assignment indicator. If subcarrier $n$ is allocated to user $k$ at RAU $m$, then $\xi_{k, n, m}=1$, else $\xi_{k, n, m}=0$. 


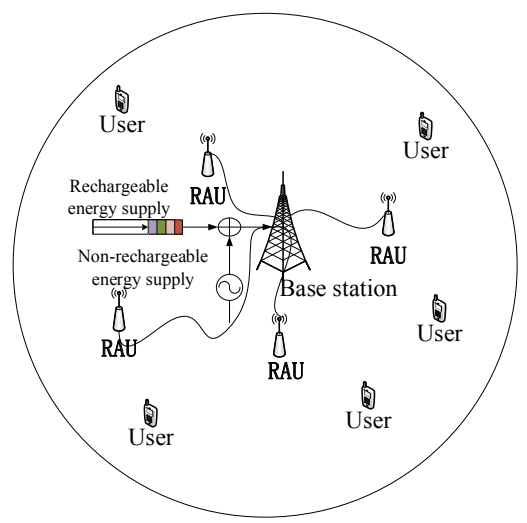

Fig. 1. A hybrid energy OFDM system with distributed antennas

To derive maximum rate-sum capacity, we assume a subcarrier is limitedly allocated to one user from one RAU [8], that is $\sum_{k} \sum_{m} \xi_{k, n, m}=1, \forall n$.

In this paper, we consider a composite channel model

$$
h_{k, n, m}=\sqrt{g_{k, m}} v_{k, n, m}
$$

where $g_{k, m}$ denotes the large scale fading coefficient that consists of path loss and shadowing between user $k$ and RAU $m$, and $v_{k, n, m}$ stands for the small scale fading between user $k$ and RAU $m$ on subcarrier $n$. We assume perfect CSI is available at the central RAU.

The large scale fading can be calculated as

$$
g_{k, m}=c s_{k, m} / d_{k, m}^{\alpha}
$$

where $c$ refers to a path gain constant at reference distance, $\alpha$ stands for path loss coefficient, $d_{k, m}$ is the distance between RAU $m$ and user $k$ and $s_{k, m}$ denotes is a shadow fading variable, which follows logarithmic normal distribution. The received SNR of user $k$ on subchannel $n$ from RAU $m$ is

$$
\gamma_{k, n, m}=p_{k, n, m}\left|h_{k, n, m}\right|^{2} / \sigma_{z}^{2}, \text { if } \xi_{k, n, m}=1
$$

\subsection{Hybrid power supply model}

As shown in Fig.1, there are two type of energy sources in the base station: the renewable energy from energy harvesting battery and the non-renewable energy of power grid or diesel engine. There are three parts of energy consumption at the base station: the power amplifier, the fiber-optical dissipation and the circuit processing including filtering, modulation, channel coding and so on. For the circuit processing and fiber-optical dissipation are also powered by the two energy resource, i.e. $P_{\mathrm{C}}=P_{\mathrm{C}}^{\mathrm{R}}+P_{\mathrm{C}}^{\mathrm{N}}$, and $(M-1) P_{F}=P_{\mathrm{F}}^{\mathrm{N}}+P_{\mathrm{F}}^{\mathrm{R}}$, where $P_{\mathrm{C}}^{\mathrm{R}}$ and $P_{\mathrm{C}}^{\mathrm{N}}$ refer to the circuit processing power supplied 
by renewable energy and non-renewable energy, respectively, $P_{\mathrm{F}}^{\mathrm{R}}$ and $P_{\mathrm{F}}^{\mathrm{N}}$ refer to the fiber-optical power supplied by renewable energy and non-renewable energy, respectively.

Similarly, for the power amplifier the transmit power is written as $\sum_{k=1}^{K} \sum_{n=1}^{N} \sum_{m=1}^{M} \xi_{k, n, m} p_{k, n, m}=P_{\mathrm{A}}^{\mathrm{R}}+P_{\mathrm{A}}^{\mathrm{N}}$, where $P_{\mathrm{A}}^{\mathrm{N}}=\sum_{k=1}^{K} \sum_{n=1}^{N} \sum_{m=1}^{M} \xi_{k, n, m} p_{k, n, m}-P_{\mathrm{A}}^{\mathrm{R}}$ and $\quad P_{\mathrm{A}}^{\mathrm{R}}$ is the power supplied by non-renewable energy and renewable energy respectively.

\section{Resource allocation}

\subsection{Problem Formulation}

Suppose the system operates in slotted time. The channel is non-time-varying during each slot, and resource allocation is performed at every slot. If continuous-rate adaptation is used, the overall date rate or the SE of user $k$ at the current time slot is given by [7],

$$
R_{k}=\frac{1}{N} \sum_{n=1}^{N} \sum_{m=1}^{M} \xi_{k, n, m} \log _{2}\left(1+\beta \gamma_{k, n, m}\right),
$$

where $\beta=-1.5 / \ln (5 B E R), 1 / \beta$ is SNR gap and $B E R$ is required bit error rate.

And we assume the weighted sum rate of the system $R$ can be calculated as

$$
R=\sum_{k=1}^{K} \theta_{k} R_{k}
$$

where $\theta_{k}$ stands for different user priority given by upper layer, and $0 \leq \theta_{k} \leq 1$.

The total non-renewable power consumption of the system is

$$
P_{\text {total }}^{\mathrm{N}}=\left(\sum_{k=1}^{K} \sum_{n=1}^{N} \sum_{m=1}^{M} \xi_{k, n, m} p_{k, n, m}-P_{\mathrm{A}}^{\mathrm{R}}\right) / \eta+P_{\mathrm{C}}^{\mathrm{N}}+P_{\mathrm{F}}^{\mathrm{N}},
$$

where $\eta$ denotes the efficiency of the power amplifier.

And in this paper, we define net ECI (J/bit) [9] of the system as

$$
W_{\mathrm{aECI}}=\frac{P_{\mathrm{mog}}^{\mathrm{N}}}{R}
$$

where the numerator is the non-renewable power of the system which costs in later utilization. Whereas, the renewable power is renewable and self-sustainable and need no extra pay.

Let $p_{m}^{\max }$ and $P_{\max }^{\mathrm{R}}$ denote the maximum output power of the power amplifier at RAU $m$ and the maximal power that the energy harvesting battery can supply. Note that $P_{\max }^{\mathrm{R}}$ is determined by both the battery capacity and the remained energy of the last time slot. We would like to minimize net ECI under the system QoS constraint. The corresponding optimization problem is formulated as 


$$
\begin{array}{lll}
\min _{\mathrm{P}, \mathrm{B}} & W_{\mathrm{nECI}}=\frac{P_{\text {total }}^{\mathrm{N}}}{R} & \\
\text { s.t. } & C 1: \sum_{k} \sum_{m} \xi_{k, n, m}=1, \forall n & \mathrm{C} 2: P_{\mathrm{C}}=P_{\mathrm{C}}^{\mathrm{R}}+P_{\mathrm{C}}^{\mathrm{N}} \\
& \mathrm{C} 3:(M-1) P_{F}=P_{\mathrm{F}}^{\mathrm{N}}+P_{\mathrm{F}}^{\mathrm{R}} & \mathrm{C} 4: P_{\mathrm{A}}^{\mathrm{R}} / \eta+P_{\mathrm{F}}^{\mathrm{R}}+P_{\mathrm{C}}^{\mathrm{R}} \leq P_{\max }^{\mathrm{R}} \\
& \mathrm{C} 5: \xi_{k, n, m}=\{0,1\}, \forall k, n, m & \mathrm{C} 6: \sum_{k=1}^{K} \sum_{n=1}^{N} \xi_{k, n, m} p_{k, n, m} \leq p_{m}^{\max }, \forall m \\
& \mathrm{C} 7: R \geq R_{\min } \quad \mathrm{C} 8: p_{k, n, m}, P_{\mathrm{A}}^{\mathrm{R}}, P_{\mathrm{F}}^{\mathrm{R}}, P_{\mathrm{C}}^{\mathrm{R}}, p^{\mathrm{R}}, P_{\mathrm{C}}^{\mathrm{N}}, P_{\mathrm{C}}^{\mathrm{R}} \geq 0, \forall k, n, m
\end{array}
$$

where the optimization variables are the power allocation policy $\mathrm{P}=\left\{P_{\mathrm{A}}^{\mathrm{R}}, P_{\mathrm{F}}^{\mathrm{R}}, P_{\mathrm{C}}^{\mathrm{R}}, p^{\mathrm{R}}, P_{\mathrm{C}}^{\mathrm{N}}, P_{\mathrm{C}}^{\mathrm{R}}, p_{k, n, m}, \forall k, n, m\right\}$ and the subcarrier assignment policy $\Xi=\left\{\xi_{k, n, m}, \forall k, n, m\right\}$, and $R_{\min }$ is the system minimum rate requirement.

The constraint $\mathrm{C} 4$ indicates that consumed renewable power should be no more than that the energy harvesting battery can supply. C6 ensures that transmit power at RAU $m$ is no more than the maximum output power of the power amplifier at RAU $m$. C1 and C5 are constraints on the subcarrier assignment, and $\mathrm{C} 1$ indicates that each subcarrier can only be allocated to one user from one RAU. C7 specifies the QoS constraints. There is a binary variable $\xi_{k, n, m}$ in (10), thus it is a $0-1$ mixed integer nonlinear problem. This kind of problem is known as NP-complete. And the objective function is a fraction and is nonconvex. The exhaustive search algorithm needs to search $(K M)^{N}$ subcarrier allocation policies to solve (10). The computational complexity increases exponentially with the number of subcarriers.

\subsection{Transformation of the optimal problem and fractional programming}

There are two steps of transformation to solve (10). First, we set $\varepsilon=\frac{P_{\text {total }}^{\mathrm{N}}}{R}$ and define

$$
y(\mathrm{P}, \Xi, \varepsilon)=P_{\text {total }}^{\mathrm{N}}-\varepsilon R
$$

And we define $Y(\varepsilon)=\min _{\mathrm{P}, \Xi} y(\mathrm{P}, \Xi, \varepsilon)$ and $\varepsilon^{*}=\min _{\mathrm{P}, \Xi} \frac{P_{\text {total }}^{\mathrm{N}}}{R}$. It has been proved in [10] that $Y(\varepsilon)$ is equivalent to the original objective function in (10) if and only if $Y\left(\varepsilon^{*}\right)=\min _{\mathrm{P}, \Xi} y\left(\mathrm{P}, \Xi, \varepsilon^{*}\right)=y\left(\mathrm{P}^{*}, \Xi^{*}, \varepsilon^{*}\right)=0$.

And then we relax the binary variable $\xi_{k, n, m}$ to a continuous variable between 0 and 1 and $\mathrm{C} 5$ is transformed to

$$
0 \leq \xi_{k, n, m} \leq 1
$$

In this paper, we solve the problem in two nest iterative algorithm. for the outer iteration, we adopt the Dinkelbach method and propose Algorithm 1 shown at Table 1. The convergence is proved in [12] if the inner iterative algorithm solves the (10). 


$$
\begin{aligned}
& \min _{\mathrm{P}, \mathrm{\Xi}} P_{\text {total }}^{\mathrm{N}}-\varepsilon R \\
& \text { s.t. } \mathrm{C} 1-\mathrm{C} 4,(11), \mathrm{C} 7-\mathrm{C} 8
\end{aligned}
$$

Table 1. Proposed Algorithm1 based on iterative Dinkelbach method

\begin{tabular}{|l|l|}
\hline \multicolumn{2}{|l|}{ Algorithm 1 Outer Iterative Algorithm } \\
\hline $1:$ & $\begin{array}{l}\text { Initialization: Set the maximize number of iteration IterNum }{ }_{\text {max }}, \text { the tolerance } \Delta, \\
\text { the initial value of } \varepsilon(0), \text { convergence }=0 .\end{array}$ \\
\hline $2:$ & Repeat \\
\hline $3:$ & $\begin{array}{l}\text { Solve the problem }(12) \text { by the inner iterative algorithm listed in Table 2 for a given } \varepsilon \\
\text { and obtain the } P_{\text {total }}^{\mathrm{N}} \text { and } R\end{array}$ \\
\hline $4:$ & If $\mid P_{\text {total }}^{\mathrm{N}}-\varepsilon($ iter $) R \mid \leq \Delta$ \\
\hline $5:$ & convergence $=1, \varepsilon^{*}=P_{\text {total }}^{\mathrm{N}}($ iter $) / R($ iter $)$ \\
\hline $6:$ & else \\
\hline $7:$ & Update $\varepsilon($ iter $)=\frac{P_{\text {total }}^{\mathrm{N}}(\text { iter })}{R(\text { iter })}$. \\
\hline $8:$ & End if \\
\hline $9:$ & Until iter $>$ IterNum $_{\text {max }}$ or convergence $=1$ \\
\hline
\end{tabular}

\subsection{Lagrange slack method and subgradient method}

To solve (12), we derive the Lagrange function by introducing non-negative Lagrange multipliers [11],

$$
\begin{aligned}
& L(\mathrm{P}, \Xi, \rho, \psi, \lambda, \boldsymbol{\mu}, \phi)=\left(\sum_{k=1}^{K} \sum_{n=1}^{N} \sum_{m=1}^{M} \xi_{k, n, m} p_{k, n, m}-P_{\mathrm{A}}^{\mathrm{R}}\right) / \eta+P_{\mathrm{C}}^{\mathrm{N}}+P_{\mathrm{F}}^{\mathrm{N}}-\varepsilon R \\
& +\rho\left(P_{\mathrm{C}}^{\mathrm{N}}+P_{\mathrm{C}}^{\mathrm{R}}-P_{\mathrm{C}}\right)+\lambda\left(P_{\mathrm{A}}^{\mathrm{R}} / \eta+P_{\mathrm{F}}^{\mathrm{R}}+P_{\mathrm{C}}^{\mathrm{R}}-P_{\text {max }}^{\mathrm{R}}\right)-\phi\left(R-R_{\min }\right) \\
& +\psi\left(P_{\mathrm{F}}^{\mathrm{N}}+P_{\mathrm{F}}^{\mathrm{R}}-(M-1) P_{\mathrm{F}}\right)+\sum_{m=1}^{M} \mu_{m}\left(\sum_{k=1}^{K} \sum_{n=1}^{N} \xi_{k, n, m} p_{k, n, m}-p_{m}^{\max }\right)
\end{aligned}
$$

where $\rho, \psi, \lambda, \boldsymbol{\mu}, \phi$ are the Lagrange multipliers of $\mathrm{C} 2-\mathrm{C} 4$ and $\mathrm{C} 6-\mathrm{C} 7$, respectively. Note that (12), C1 and C8 are used for later derivation for the optimal solution. The corresponding Dual problem of (13) is omitted due to spacing limit. With the KKT conditions, we can obtain

$$
p_{k, n, m}^{*}=\left[\frac{\theta_{k}(\varepsilon+\phi)}{N \ln 2\left(1 / \eta+\mu_{m}\right)}-\frac{\sigma_{z}^{2}}{\beta\left|h_{k, n, m}\right|^{2}}\right]^{+}
$$

where the notation $[x]^{+}$represents $[x]^{+}=\max \{0, x\}$.

To determine the subcarrier assignment scheme, we differentiate the Lagrange function with respect to $\xi_{k, n, m}$, we can obtain

$$
\partial L(\ldots) / \partial \xi_{k, n, m}=\left(1 / \eta+\mu_{m}\right) p_{k, n, m}-\theta_{k}(\varepsilon+\phi) \log _{2}\left(1+\beta p_{k, n, m}\left|h_{k, n, m}\right|^{2} / \sigma_{z}^{2}\right) / N
$$


It can be observed from (16) that $L(\mathrm{P}, \Xi, \rho, \psi, \lambda, \boldsymbol{\mu}, \mathbf{v}, \phi)$ is an affine function in $\xi_{k, n, m}$. Hence to obtain the minimum value of $L(\mathrm{P}, \Xi, \rho, \psi, \lambda, \boldsymbol{\mu}, \phi)$ with given Lagrange multipliers and in consideration of $\mathrm{C} 5$, the optimal $\xi_{k, n, m}^{*}$ is given as

$$
\xi_{i, n, j}^{*}=\left\{\begin{array}{l}
1, \text { if }(i, j)=\arg \min _{(k, m)} \partial L(\mathrm{P}, \Xi, \alpha, \beta, \gamma, \lambda, \boldsymbol{\mu}) / \partial \xi_{k, n, m} \\
0, \text { else. }
\end{array}\right.
$$

Similarly, it can be derived that $L(\mathrm{P}, \Xi, \rho, \psi, \lambda, \boldsymbol{\mu}, \mathbf{v}, \phi)$ is an affine function in $P_{\mathrm{A}}^{\mathrm{R}}, P_{\mathrm{F}}^{\mathrm{R}}, P_{\mathrm{C}}^{\mathrm{R}}, p^{\mathrm{R}}, P_{\mathrm{C}}^{\mathrm{N}}, P_{\mathrm{C}}^{\mathrm{R}}$. And to minimize the consumption of non-renewable energy, we allocate the power as

$$
\begin{gathered}
P_{\mathrm{A}}^{\mathrm{R}}=\min \left(\sum_{k=1}^{K} \sum_{n=1}^{N} \sum_{m=1}^{M} \xi_{k, n, m} p_{k, n, m},\left[\eta P_{\max }^{\mathrm{R}}\right]^{+}\right) \\
P_{\mathrm{C}}^{\mathrm{R}}=\min \left(P_{\mathrm{C}},\left[P_{\text {max }}^{\mathrm{R}}-P_{\mathrm{A}}^{\mathrm{R}} / \eta\right]^{+}\right) \\
P_{\mathrm{C}}^{\mathrm{N}}=\left[P_{\mathrm{C}}-P_{\mathrm{C}}^{\mathrm{R}}\right]^{+} \\
P_{\mathrm{F}}^{\mathrm{R}}=\min \left((M-1) P_{\mathrm{F}},\left[P_{\text {max }}^{\mathrm{R}}-P_{\mathrm{A}}^{\mathrm{R}} / \eta-P_{\mathrm{C}}^{\mathrm{R}}\right]^{+}\right) \\
P_{\mathrm{F}}^{\mathrm{N}}=\left[(M-1) P_{\mathrm{F}}-P_{\mathrm{F}}^{\mathrm{R}}\right]^{+}
\end{gathered}
$$

After allocating the power and subcarrier, we update the Lagrange multipliers using the subgradient method to guarantee convergence. The update equations are given by

$$
\begin{gathered}
\lambda^{i+1}=\left[\lambda^{i}+\varsigma_{1}^{i}\left(P_{\mathrm{A}}^{\mathrm{R}} / \eta+P_{\mathrm{F}}^{\mathrm{R}}+P_{\mathrm{C}}^{\mathrm{R}}-P_{\max }^{\mathrm{R}}\right)\right]^{+} \\
\mu_{m}^{i+1}=\left[\mu_{m}^{i}+\varsigma_{2}^{i}\left(\sum_{k=1}^{K} \sum_{n=1}^{N} \xi_{k, n, m} p_{k, n, m}-p_{m}^{\max }\right)\right]^{+} \\
\phi^{i+1}=\left[\phi^{i}-\varsigma_{3}^{i}\left(R-R_{\min }\right)\right]^{+}
\end{gathered}
$$

where $i$ represents the iteration number, and $\zeta$ are the positive iterative step size.

Note that there is no need to update $\rho$ and $\psi$ since $\mathrm{C} 2$ and $\mathrm{C} 4$ are equality constraints. If the step size satisfies the infinite travel condition [12], the above update equations guarantee the convergence of optimal solution. Based on the analysis, we propose Algorithm 2 to solve the problem (13), which is shown at Table 2. 
Table 2. Proposed Algorithm 2

\begin{tabular}{|l|l|}
\hline Algorithm 2 Inner Iterative Algorithm \\
\hline $1:$ & $\begin{array}{l}\text { Initialization: Set the maximize number of iteration } I_{m a x}, \text { the tolerance } \Delta, \text { the } \\
\text { initial value of Lagrange multipliers, convergence }=0 .\end{array}$ \\
\hline $2:$ & Repeat \\
\hline $3:$ & Calculate $\quad p_{k, n, m}, \forall k, n, m$ according to $(14)$. \\
\hline $4:$ & Calculate $\partial L(\mathrm{P}, \Xi, \rho, \psi, \lambda, \boldsymbol{\mu}, \phi) / \partial \xi_{k, n, m}, \forall k, m$ according to $(15)$. \\
\hline $5:$ & Obtain subcarrier assignment $\xi_{k, n, m}$ according to $(16)$. \\
\hline $6:$ & Allocate power $P_{\mathrm{A}}^{\mathrm{R}}, P_{\mathrm{F}}^{\mathrm{R}}, P_{\mathrm{C}}^{\mathrm{R}}, p^{\mathrm{R}}, P_{\mathrm{C}}^{\mathrm{N}}, P_{\mathrm{C}}^{\mathrm{R}}$ according to $(17-21)$. \\
\hline $7:$ & Update: Lagrange multipliers $\psi(i), \lambda(i), \boldsymbol{\mu}(i)$, according to $(22-24)$. \\
\hline $8:$ & Calculate $P_{\text {total }}^{\mathrm{N}}(i)-\varepsilon R(i)$ \\
\hline $9:$ & If $\mid\left(\left(P_{\text {total }}^{\mathrm{N}}(i)-\varepsilon R(i)\right)-\left(P_{\text {total }}^{\mathrm{N}}(i-1)-\varepsilon R(i-1)\right)\right.$ \\
\hline 10: & convergence $=1$. \\
\hline $11:$ & End if \\
\hline $12:$ & Until $i>I_{\text {max }}$ or convergence $=1$ \\
\hline
\end{tabular}

\section{Simulation results and discussion}

In this section, we evaluate the performance of the proposed algorithm. The OFDM system has $N=128$ subcarrier, $K$ users and $M$ RAUs. Users are located within a radius $D=1000$ meters from the base station, and the locations is generated randomly obeying uniformly distributed during the simulation.

There are three locations of RAUs, $\{(0,0)\},\{(0,0),(0, D / 2),(0,-D / 2)\}$, $\{(0,0),(0, D / 2),(0,-D / 2),(D / 2,0),(-D / 2,0)\}$. The path loss exponent $\alpha=4$ and a $8 \mathrm{~dB}$ shadowing fading coefficient is used. The small scale fading is assumed to be independent and identical distributed (i.i.d.) Reyleigh random variables. The noise variance is $\sigma_{z}^{2}=104$ $\mathrm{dBm}$. The circuit power of the base station is $P_{\mathrm{C}}=40 \mathrm{dBm}$ and the fiber optical power is $P_{\mathrm{F}}=-0.6 \mathrm{dBm}$. The maximum power of power amplifier of RAU $\mathrm{m}$ is $p_{\max }^{\mathrm{m}}=30 \mathrm{dBm}$, $\forall m$. The minimum rate requirement of system is $R_{\min }=1 \mathrm{bit} / \mathrm{s} / \mathrm{Hz}$. And $\theta_{k}=1, \forall k$. The efficiency of power amplifier is $\eta=0.38$. Because channel fading and locations of users are changing, all results are an average of 2000 simulations.
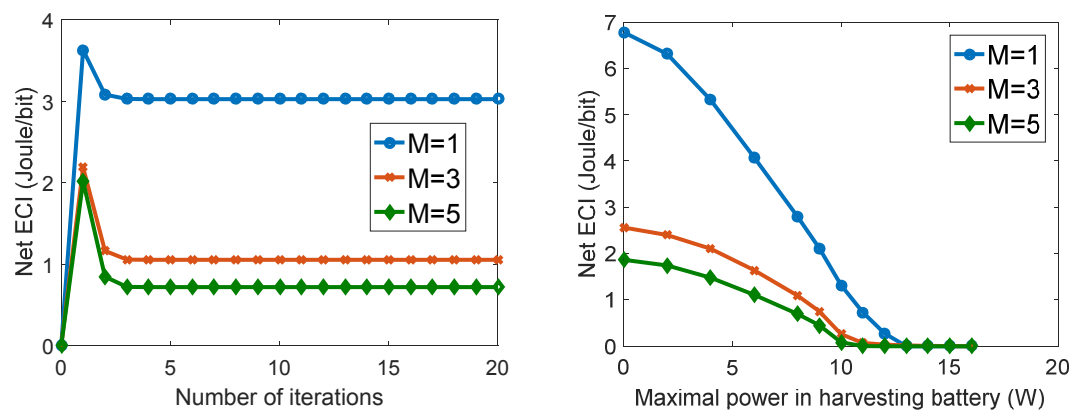
Fig. 2. Convergence of Algorithm 1 $\mathrm{N}=128, \mathrm{~K}=5$ )

Fig. 2 shows the convergence of the proposed algorithm via the number of iterations. We choose three number of RAUs, and $M=1$ refers to the OFDM-CAS system. As shown in Fig.2, the algorithm obtains a convergent solution within 4 iterations in all three scenarios. Moreover, the net ECI gets smaller in OFDMA-DAS than OFDMA-CAS system.

Fig. 3 shows the system net ECI with different value of maximum renewable power. It is reasonable that net ECI decreases as the value of maximum renewable power increases with different number of RAUs. This phenomenon shows net ECI is improved with the energy harvesting supply.
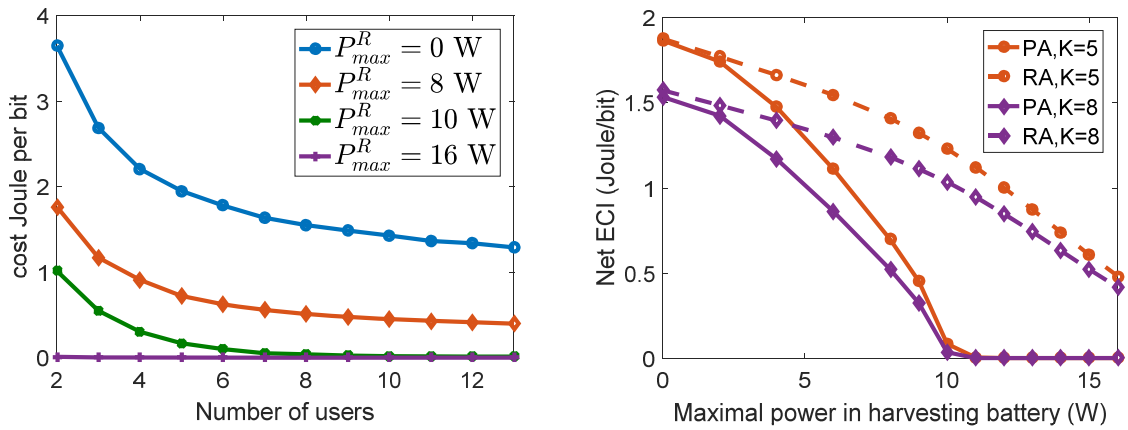

Fig. 4. Net ECI with different number of users, $\mathrm{M}=5$

Fig. 5. Comparison with RA algorithm in system net ECI

Fig. 4 shows the system net ECI with different number of users. We choose three scenarios, no renewable energy scenario, lacking renewable energy scenario and sufficient renewable energy scenario. Interestingly, the net ECI decreases with the number of users increases. And as the number of users increasing larger, the decline of net ECI gets flat. With more users, RAUs have larger probability to access subcarriers in good channel state with the fix system QoS constraint, resulting in better performance.

Fig. 5 illustrates the comparison of the proposed algorithm(PA) and the rate adaptive (RA) algorithm in system net ECI. The RA algorithm which maximize the sum rate of the system can be adopted by traditional water-filling algorithm. The proposed algorithm can achieve better performance than the RA algorithm and the improvement increases as the value of maximum renewable power increases.

\section{Conclusion}

We studied the resource allocation in a downlink OFDM DAS system with hybrid power supply. To minimize net ECI of the system with system QoS and BER requirement constraints, we proposed two nested algorithm based on fractional programming and Lagrange relaxation method. Through simulation studies, net ECI of the system under the proposed scheme was investigated and the results showed that the proposed algorithm converges quickly and improves the system net ECI significantly. Interests for future work include the transmit scheme with imperfect CSI, as well as optimization algorithm with low computational complexity.

Acknowledgments: This work was supported in part by National Natural Science Foundation of China (NSFC) (Grant Nos. 61571120, 61501113), and by Natural Science Foundation of Jiangsu Province (Grant Nos. BK20151415, BK20150630). 


\section{References}

1. Klein T E, Richard P.: ICT Energy Challenges, Impact and Solutions[C] In:19th International ICIN Conference-Innovations in Clouds, Internet and Networks (2016)

2. $\mathrm{Ku}, \mathrm{M} . \mathrm{L} ., \mathrm{Li}, \mathrm{W} ., \mathrm{Chen}, \mathrm{Y}$, Liu, K. R.: Advances in Energy Harvesting Communications: Past, Present, and Future Challenges. IEEE Communications Surveys and Tutorials 18(2): 1384--1412 (2016)

3. Ni, X., Yuan, D., Teng, Y., Song, M.: Energy efficient power allocation scheme for multi-cell with hybrid energy sources. In: Personal, Indoor, and Mobile Radio Communications (PIMRC), 2015 IEEE 26th Annual International Symposium on, pp. 1611--1616. IEEE. (2015)

4. Ng, D. W. K., Lo, E. S., Schober, R.: Energy-efficient resource allocation in OFDMA systems with hybrid energy harvesting base station. IEEE Transactions on Wireless Communications, 12(7), 3412--3427 (2013)

5. Yu, B., Ma, P., Ma, Y.: Resource allocation and energy management in ofdm-based cellular systems. In: Communication Systems (ICCS), 2016 IEEE International Conference on. IEEE, pp. 1--6. IEEE. (2016)

6. Li, J., Wang, D., Zhu, P., You, X.: Spectral efficiency analysis of single-cell multi-user large-scale distributed antenna system. IET Communications, 8(12), 2213-2221 (2014)

7. He, C., Li, G. Y., Zheng, F. C., You, X.: Energy-efficient resource allocation in OFDM systems with distributed antennas. IEEE Transactions on Vehicular Technology, 63(3), 1223-1231 (2014)

8. Xu, W., Niu, K., He, Z., Wu, W.: Resource allocation in multiuser OFDM distributed antenna systems. In: Vehicular Technology Conference, 2007. VTC-2007 Fall. 2007 IEEE $66^{\text {th }}$, pp. 1797-1801. IEEE. (2007)

9. Auer, G., Blume, O., Giannini, V., Godor, I., Imran, M., Jading, Y., ... Wajda, W.: D2. 3: Energy efficiency analysis of the reference systems, areas of improvements and target breakdown. EARTH, 20(10). (2010)

10. Dinkelbach, W.: On nonlinear fractional programming. Management science, 13(7), 492-498 (1967)

11. Boyd, S., Vandenberghe, L.: Convex optimization. Cambridge university press (2004)

12. Boyd, S., Mutapcic, A.: Subgradient methods. Lecture notes of EE364b, Stanford University, Winter Quarter, 2007 (2006) 